\title{
Studies on mosquitoes (Diptera: Culicidae) and anthropic environment. 8- Survey of adult behaviour of Spissipes Section species of Culex (Melanoconion) in South-Eastern Brazil
}

\author{
Oswaldo Paulo Forattini, Maria Anice Mureb Sallum, Iná Kakitani, Eduardo Massad, \\ Daniel Marucci
}

Department of Epidemiology, School of Public Health, University of S. Paulo. Taxonomic and Systematic Research in Medical Entomology Unit of the University of S. Paulo/(NUPTEM) - Brazil

(O.P.F., M.A.M.S., I.K., D.M.)

Department of Pathology, School of Medicine, University of S. Paulo, - Brazil (E.M.)

\begin{abstract}
A study of Spissipes Section of Culex (Melanoconion) adults behaviour was carried out from August 1992 through December 1993 in human dominated (anthropic) environment in the Ribeira Valley, S.Paulo State, Brazil. By sampllng at several sites it the dominance of Culex ribeirensis and $C x$. sacchettae became evident even through a total number of ten species was recorded. Those two mosquitoes showed a clear tendency to frequent the domiciliary environment where they were caught, both indoor and outdoor, through the use of the human bait. In the outside environments, the residual patchy forests seems to display a concentration role, from which these adults spread to the open land and reach the dwellings. As their vector competence has been demonstrated through the virus isolations in natural conditions, it is advisable to pay attention to the presence of these mosquitces in the man-made environment.
\end{abstract}

Culicidae. Ecology, vectors. Culex.

Introduction

As it is well known Culex (Melanoconion) are noctumal mosquitoes of the Americas, inhabiting mainly forest environments and most active at dusk and dawn. They feed on the biood of vertebrates including humans, when available. Their competence to transmit various arboviruses has been demonstrated both through isolations from naturally infected mosquitoes and laboratory transmission trials. Thus, they are considered as important vectors of arboviruses in their sylvan natural cycle, specially the group of the Spissipes Section. Among these agents, the encephalitis viruses transmission has been seen as deserving of special treatment.
The southern geographical distribution of Melanoconion mosquitoes corresponds to the southern Brazil and northem Argentina. As far as is known, the Spissipes Section is there represented by eleven widely species spread. Five of these were found naturally infected with arboviruses. In Argentina, several bunyaviruses have been isolated from Culex delpontei and $C x$. ocossa besides alphaviruses represented by strains of the Venezuelan equine encephalitis (VEE) and the western equine encephalitis (WEE) viruses (Calisher ${ }^{3}$, 1985; Mitchell et al.20.21, 1985, 1987). In S. Paulo State, Southern Brazil, strains of VEE and a number of bunyaviruses have been isolated from $C x$. ribeirensis (formerly confounded with $C x$. crybda and $C x$. epanastasis), $C x$. sacchettae

\footnotetext{
* Research supported by "Fundaçäo de Amparo à Pesquisa do Estado de Săo Paulo (FAPESP)" (Grant no. 90/3371-6). Reprints: Oswaldo Paulo Forattini - Department of Epidemiology, School of Public Health. Taxonomic and Systematic Research in Medical Entomology Unit of the University of S.Paulo (NUPTEM)-Av. Dr. Arnaldo, 715 - 01246-904 - S.Paulo, SP-BraziI Fax: (011)282-1898

The publication of this article was supported by FAPESP (Process $9410500-0$ ). Received in 1.10.1995. Approved in 3.8.1995.
} 
and $C$ ulex sp.VR (hitherto misidentified as $C x$. taeniopus) (Calisher et al.1.2, 1982, 1983; Sallum $\left.{ }^{24}, 1994\right)$. Thus, the proportion of the isolations from these Melanoconion mosquitoes presages the incrimination species of that Section as important vectors of arboviruses.

During the undertaking of an investigation program in the Ribeira Valley region of S. Paulo State, mosquito behaviour was observed. In that area an encephalitis epidemic started earlier in 1975-1976 thereafter decreasing gradually until 1983 (Iversson ${ }^{19}, 1988$ ). Among others, mosquitoes belonging to the species referred above were focused mainly as regards their biting activities, peridomiciliary habits and its occurrence in primitive and residual woods in an anthropic environment (Forattini et al ${ }^{4.56 .69: 06}, 1981,1986,1990$. 1991). The results of these observations were that $C x$. ribeirensis and $C x$, sacchettae appeared to be widespread in the region while $C x$, delpontei, $C x$. ocossa and $C$ ulex VR appeared to the limited to certain geographical areas, at least where they were found in larger numbers. Nevertheless with regard to the general anthropic and the particular dwelling environments, the two former species seems better adapted and quite attracted to human bait.

During the 1992-1993 period, a continuation of the above-mentioned investigation program, adult mosquito collections were performed through the use of human bait and the Shannon type traps. Considering the epidemiological importance of the Culex (Melanoconion) species the results of the research will be presented in this paper for the purpose of contributing to knowledge about their biting behavior in the anthropic environment.

\section{Study areas}

The data here reported are the results of sampling carried out at several sites on the Experimental Station (ES) and at the "Galileia Farm" (GA) of the Pariquera-Açu County, S. Paulo State, Brazil, which were already described elsewhere (Forattini et al.12.15, 1993, 1994). According to the criteria adopted to mapping world ecosystems these sites were recognized as corresponding to disturbed environments (Hannah et al. ${ }^{18}$, 1994). The ES was included in the human dominated (anthropic) type where the agriculture is permanent and prevails over the primary vegetation that remains as limited remnants patches. As regards the GA environment, it was defined as partially disturbed, with secondary vegetation without intensive agricultural activity or any artificial irrigation system. The domiciliary environment was represented by the rural dwellings of the "Sitio Barra do Capinzal" (BC) that is included within the ES area.

\section{Material and Method}

Sampling was done in accordance with the procedures described elsewhere (Forattini et al. ${ }^{15}$, 1994). Thus Shannon traps operated by a two-man team were used fortnightly from 17:00 to 20:00 hours at ES and GA sites, as follows:

\section{Period Site Place Shannon trap \\ January - ES remnant forest A Shan 1 \\ December 1993

$\begin{array}{ll}\text { remnant forest B } & \text { Shan } 2 \\ \text { rice-paddy margin } & \\ \text { (open land) } & \text { Shan E }\end{array}$ \\ August 1992 - GA margin of \\ December 1993 Pariquera-Açu river Shan GA}

As Shan 1 and Shan 2 traps operated alternat, the total number of mosquitoes caught corresponded to the fortnightly rhythm.

Sampling the biting mosquitoes catches made in the domiciliary environment was done using human bait. Two collectors volunteers vacinated against yellow fever caught the females as they came to feed and alighted on their exposed legs during two hour crepuscular period, from 17:30 to $19: 30$ or from 18:00 to 20:00 hours according to :he season (Forattini et al.411.15, 1981, 1993, 1994). The collections were simultaneously performed indoors and outdoors, by one man in each place using a battery. operated aspirators to catch landing/biting females. Catches were made at $\mathrm{BC}$ from August 1992 through December 1993, following the fortnightly rhythm in a regular manner. The biting activity was estimated by the Williams media $\left(\overline{\mathbf{x}}_{\mathrm{w}}\right.$ was originally used (Haddow ${ }^{16.17}$, 1954, 1960).

Regarding the degree of domiciliation, the synanthropic index of Nuorteva was calculated for both species, according to the percentages of specimens collected at the different places. In this manner an estimation of the synanthropy of these mosquitoes was proposed (Nuorteva ${ }^{22}, 1963$; Povolny 23, 1971: Forattini et al. ${ }^{12}, 1993$ ). For this 
purpose the various collection places were considered as follows:

$$
S=\frac{2 a+b-2 c}{2}
$$

Where:

$\mathrm{a}=$ percentage collected in the domiciliary environment $(\mathrm{BC})$.

$\mathrm{b}=$ percentage collected in the anthropic and partially disturbed environments (Shan E and Shan GA).

$\mathrm{c}=$ percentage collected in the remnant patchy forest environments (Shan 1 and Shan 2).

$s=$ Nuorteva index .

Thus the comparisons were undertaken within the disturbed anthropic environment and covered its various aspects here studied. To obtain a general over-view of $C x$. ribeirensis's and $C x$, sacchettae's degrees of synanthropy, the relations between the total numbers of specimens collected at each of the places referred to above were calculated as synanthropic ratios (sr).

\section{Results}

A total number of 6,631 adult mosquitoes $(6,344$ females and 287 males) of the Spissipes Section was collected. Of that number 5,602 $(84.5 \%)$ were caught with the Shannon traps whereas $764(11.5 \%)$ with the use of human bait. Ten species were obtained and their specific distribution was as follows:

\begin{tabular}{|c|c|}
\hline Species & $N$ \\
\hline Cx. delpontei & 9 \\
\hline Cx. faurani & 17 \\
\hline Cx. lopesi & 1 \\
\hline Cx. ocossa & 139 \\
\hline Cx. pedroi & 33 \\
\hline Cx. pereyrai & 1 \\
\hline Cx. ribeirensis & 3.093 \\
\hline Cx. sacchettae & 3,266 \\
\hline Cx. spissipes & 22 \\
\hline CulexvR & 50 \\
\hline Total & 6,63 \\
\hline
\end{tabular}

(*) less than 0.1

The results are presented, in accordance with the several samples obtained at the ES and GA sites, and the methods employed, in Table 1. Thus $C x$. ribeirensis and $C x$. sacchettae jointly accounted for $95.9 \%$ of the Spissipes Section specimens collected. The behavior of these two species called for particular attention in the present paper for that reason.

Shannon traps.- The monthly distributions of $C x$. ribeirensis and $C x$. sacchettae are presented in Tables 2 and 3 . Only females were obtained by the Shan GA. Considering the agricultural anthropic environment of the ES area it seems that those mosquitoes were mainly produced in the months of February-April when a total of $74.6 \%$ of the adults were caught. However, as a general feature, the remnant forest patches (Shan I and Shan 2) produced

Tabela 1 - Number of adult mosquitoes of the Spissipes Section of Culex (Melanoconion) collected in the anthropic (ES) and partially disturbed environments (GA) August 1992 through December 1993.

\begin{tabular}{|c|c|c|c|c|c|c|c|c|c|c|c|c|c|c|c|}
\hline \multirow{3}{*}{ Species } & \multicolumn{4}{|c|}{ ES remnant forest } & \multirow{2}{*}{\multicolumn{2}{|c|}{$\frac{\text { ES open land }}{\text { Shan } E}$}} & \multicolumn{4}{|c|}{ BC dwellings ( $h b)$} & \multicolumn{2}{|c|}{$\mathrm{GA}$} & & & \\
\hline & \multicolumn{2}{|c|}{ Shan 1} & \multicolumn{2}{|c|}{ Shan 2} & & & \multicolumn{2}{|c|}{ Outdoor } & \multicolumn{2}{|c|}{ Indoor } & \multicolumn{2}{|c|}{ Shan GA } & \multicolumn{3}{|c|}{ Total } \\
\hline & $f$ & $\mathrm{~m}$ & $\uparrow$ & $\mathrm{m}$ & $f$ & $\mathrm{~m}$ & $f$ & $m$ & $f$ & $\mathrm{~m}$ & $\uparrow$ & $\mathrm{m}$ & $f$ & $\mathrm{~m}$ & $\mathrm{~T}$ \\
\hline Cx. delpontei & 1 & - & - & 1 & 5 & $a$ & - & . & - & . & - & - & 6 & 3 & 9 \\
\hline$C x$ & 2 & 12 & 2 & 1 & 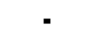 & - & - & 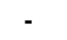 & - & - & - & - & 4 & 13 & 17 \\
\hline$C \times$. & - & 1 & - & - & - & - & - & 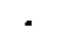 & - & - & - & - & - & 1 & 1 \\
\hline$C x$ & 23 & - & 2 & - & 102 & 7 & 3 & 1 & - & . & 1 & - & 131 & 8 & 139 \\
\hline$C x . P$ & 3 & 25 & 2 & 3 & - & - & . & - & - & - & - & - & 5 & 28 & 33 \\
\hline Cx. pereyrai & - & 1 & - & - & - & - & - & - & - & - & - & - & - & 1 & 1 \\
\hline girensis & 1,436 & 63 & 533 & 22 & 403 & 4 & 134 & 1 & 92 & - & 405 & - & 3,003 & 90 & 3,093 \\
\hline chettae & $1, t 13$ & 59 & 979 & 78 & 338 & 5 & 274 & - & 258 & - & 162 & - & 3,124 & 142 & 3,266 \\
\hline$C x$. & 14 & - & 7 & 1 & - & 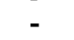 & - & - & - & - & - & . & 21 & 1 & 22 \\
\hline Cx. V & 19 & - & 29 & - & - & - & 1 & $\cdot$ & $\cdot$ & $\cdot$ & 1 & - & 50 & 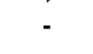 & 50 \\
\hline Total & 2,611 & 161 & 1,554 & 106 & 848 & 18 & 412 & 2 & 350 & - & 569 & - & 6,344 & 287 & 6,631 \\
\hline
\end{tabular}


Table 2 - Monthly distribution of Culex ribeirensis and $C x$. sachettae collected in Shannon traps in the anthropic environment (ES) area, January through December 1993.

\begin{tabular}{|c|c|c|c|c|c|c|c|c|c|c|c|c|c|c|c|}
\hline \multirow{3}{*}{ Monthy } & \multirow{3}{*}{ Shan } & \multicolumn{4}{|c|}{ Cx. ribeirensis } & \multicolumn{4}{|c|}{ Cx. sacchettae } & \multicolumn{6}{|c|}{ Total } \\
\hline & & \multicolumn{2}{|c|}{ female } & \multicolumn{2}{|c|}{ male } & \multicolumn{2}{|c|}{ fernale } & \multicolumn{2}{|c|}{ male } & \multicolumn{2}{|c|}{ female } & \multicolumn{2}{|c|}{ male } & \multicolumn{2}{|c|}{$\mathrm{T}$} \\
\hline & & $n$ & $\%$ & $\mathrm{n}$ & $\%$ & $n$ & $\%$ & $n$ & $\%$ & $n$ & $\%$ & $n$ & $\%$ & & \\
\hline January & $\begin{array}{l}1 \\
2 \\
E\end{array}$ & $\begin{array}{l}15 \\
54 \\
34\end{array}$ & $\begin{array}{l}0.6 \\
2.3 \\
1.4\end{array}$ & $\begin{array}{l}- \\
1 \\
-\end{array}$ & -1.1 & $\begin{array}{c}28 \\
13 \\
6\end{array}$ & $\begin{array}{l}1.1 \\
0.5 \\
0.2\end{array}$ & $\begin{array}{l}- \\
2 \\
-\end{array}$ & $\begin{array}{l}- \\
1.4 \\
-\end{array}$ & $\begin{array}{l}43 \\
67 \\
40\end{array}$ & $\begin{array}{l}0.9 \\
1.4 \\
0.8\end{array}$ & 3 & $\begin{array}{l}- \\
-\end{array}$ & $\begin{array}{l}43 \\
70 \\
40\end{array}$ & $\begin{array}{l}0.9 \\
1.4 \\
0.8\end{array}$ \\
\hline February & $\begin{array}{l}1 \\
2 \\
E\end{array}$ & $\begin{array}{c}659 \\
103 \\
3\end{array}$ & $\begin{array}{r}27.8 \\
4.3 \\
0.1\end{array}$ & $\begin{array}{c}41 \\
3 \\
-\end{array}$ & $\begin{array}{r}46.0 \\
3.4 \\
-\end{array}$ & $\begin{array}{c}97 \\
14 \\
2\end{array}$ & $\begin{array}{l}4.0 \\
0.6 \\
0.1\end{array}$ & $\begin{array}{l}5 \\
3 \\
-\end{array}$ & $\begin{array}{l}3.5 \\
2.1 \\
.\end{array}$ & $\begin{array}{c}756 \\
117 \\
5\end{array}$ & $\begin{array}{r}15.7 \\
2.4 \\
0.1\end{array}$ & $\begin{array}{c}46 \\
6 \\
-\end{array}$ & $\begin{array}{r}19.9 \\
2.6 \\
.\end{array}$ & $\begin{array}{c}802 \\
123 \\
5\end{array}$ & $\begin{array}{r}15.9 \\
2.4 \\
0.1\end{array}$ \\
\hline March & $\begin{array}{l}1 \\
2 \\
E\end{array}$ & $\begin{array}{l}313 \\
222 \\
152\end{array}$ & $\begin{array}{r}13.2 \\
9.3 \\
6.4\end{array}$ & $\begin{array}{c}18 \\
11 \\
4\end{array}$ & $\begin{array}{r}20.2 \\
12.3 \\
4.5\end{array}$ & $\begin{array}{l}237 \\
273 \\
241\end{array}$ & $\begin{array}{r}9.7 \\
15.3 \\
9.9\end{array}$ & $\begin{array}{c}30 \\
42 \\
2\end{array}$ & $\begin{array}{r}21.1 \\
29.6 \\
1.4\end{array}$ & $\begin{array}{l}550 \\
595 \\
393\end{array}$ & $\begin{array}{r}11.4 \\
12.4 \\
8.2\end{array}$ & $\begin{array}{c}48 \\
53 \\
6\end{array}$ & $\begin{array}{r}20.8 \\
22.9 \\
2.6\end{array}$ & $\begin{array}{l}598 \\
648 \\
399\end{array}$ & $\begin{array}{r}11.9 \\
12.9 \\
7.9\end{array}$ \\
\hline April & $\begin{array}{l}1 \\
2 \\
\mathrm{E}\end{array}$ & $\begin{array}{c}149 \\
57 \\
32\end{array}$ & $\begin{array}{l}6.3 \\
2.4 \\
1.3\end{array}$ & : & $\begin{array}{l}- \\
3.4 \\
-\end{array}$ & $\begin{array}{c}598 \\
289 \\
25\end{array}$ & $\begin{array}{r}24.6 \\
11.9 \\
1.0\end{array}$ & $\begin{array}{c}16 \\
13 \\
-\end{array}$ & $\begin{array}{c}11.3 \\
9.1 \\
-\end{array}$ & $\begin{array}{c}747 \\
346 \\
57\end{array}$ & $\begin{array}{r}15.6 \\
7.2 \\
1.2\end{array}$ & $\begin{array}{c}16 \\
16 \\
-\end{array}$ & $\begin{array}{l}6.9 \\
6.9 \\
-\end{array}$ & $\begin{array}{c}763 \\
362 \\
57\end{array}$ & $\begin{array}{r}15.2 \\
7.2 \\
1.1\end{array}$ \\
\hline May & $\begin{array}{l}1 \\
2 \\
\mathrm{E}\end{array}$ & $\begin{array}{l}66 \\
13 \\
21\end{array}$ & $\begin{array}{l}2.8 \\
0.5 \\
0.9\end{array}$ & $\begin{array}{l}- \\
1\end{array}$ & 1.1 & $\begin{array}{c}96 \\
142 \\
8\end{array}$ & $\begin{array}{l}3.9 \\
5.8 \\
0.3\end{array}$ & $\begin{array}{l}3 \\
5 \\
-\end{array}$ & $\begin{array}{l}2.1 \\
3.5 \\
.\end{array}$ & $\begin{array}{c}162 \\
155 \\
29\end{array}$ & $\begin{array}{l}3.4 \\
3.2 \\
0.6\end{array}$ & $\begin{array}{l}3 \\
6 \\
-\end{array}$ & $\begin{array}{l}1.3 \\
2.6 \\
\cdot\end{array}$ & $\begin{array}{c}165 \\
161 \\
29\end{array}$ & $\begin{array}{l}3.3 \\
3.2 \\
0.6\end{array}$ \\
\hline June & $\begin{array}{l}1 \\
2\end{array}$ & $\begin{array}{l}9 \\
1\end{array}$ & $\begin{array}{c}0.4 \\
\ldots\end{array}$ & - & - & $:$ & $=$ & - & - & 9 & 0.2 & - & - & 9 & 0.2 \\
\hline & $E$ & 29 & 1.2 & - & - & 9 & 0.4 & - & - & 38 & 0.8 & - & . & 38 & 0.8 \\
\hline July & $\begin{array}{l}1 \\
2 \\
E\end{array}$ & $\begin{array}{c}67 \\
3 \\
2 ?\end{array}$ & $\begin{array}{l}2.8 \\
0.1 \\
0.9\end{array}$ & $\begin{array}{l}1 \\
-\end{array}$ & $\begin{array}{l}1.1 \\
:\end{array}$ & $\begin{array}{l}9 \\
-\end{array}$ & $\begin{array}{l}0.4 \\
0.2\end{array}$ & : & $\begin{array}{l}1.4 \\
- \\
-\end{array}$ & $\begin{array}{c}76 \\
3 \\
26\end{array}$ & $\begin{array}{l}1.6 \\
\ldots .5\end{array}$ & $\begin{array}{l}3 \\
-\end{array}$ & $\begin{array}{l}1.3 \\
-\end{array}$ & $\begin{array}{c}79 \\
3 \\
26\end{array}$ & $\begin{array}{l}1.6 \\
0.1 \\
0.5\end{array}$ \\
\hline August & $\begin{array}{l}E \\
1 \\
2\end{array}$ & $\begin{array}{c}22 \\
19 \\
5\end{array}$ & $\begin{array}{l}0.9 \\
0.8 \\
0.2\end{array}$ & $i$ & $\begin{array}{l}- \\
1.1\end{array}$ & $\begin{array}{l}4 \\
7 \\
2\end{array}$ & $\begin{array}{l}0.2 \\
0.3 \\
0.1\end{array}$ & i & i & $\begin{array}{l}26 \\
26 \\
7\end{array}$ & $\begin{array}{l}0.5 \\
0.5 \\
0.1\end{array}$ & $\begin{array}{l}- \\
\end{array}$ & $\begin{array}{l}- \\
- \\
0.4\end{array}$ & $\begin{array}{c}26 \\
26 \\
8\end{array}$ & $\begin{array}{l}0.5 \\
0.5 \\
0.2\end{array}$ \\
\hline & $E$ & 1 & & - & - & - & - & - & - & 1 & & - & - & 1 & $\cdots$ \\
\hline September & $\begin{array}{l}1 \\
2 \\
E\end{array}$ & $\begin{array}{c}51 \\
24 \\
1\end{array}$ & $\begin{array}{l}2.1 \\
1.0\end{array}$ & $\begin{array}{l}2 \\
- \\
.\end{array}$ & $\begin{array}{l}2.2 \\
- \\
-\end{array}$ & $\begin{array}{c}14 \\
109 \\
-\end{array}$ & $\begin{array}{l}0.6 \\
4.5 \\
-\end{array}$ & 10 & 7.0 & $\begin{array}{c}65 \\
133 \\
1\end{array}$ & $\begin{array}{l}1.3 \\
2.8\end{array}$ & $\begin{array}{c}2 \\
10 \\
\end{array}$ & $\begin{array}{l}0.9 \\
4.3 \\
.\end{array}$ & $\begin{array}{c}67 \\
143 \\
1\end{array}$ & $\begin{array}{l}1.3 \\
2.8\end{array}$ \\
\hline October & $\begin{array}{l}1 \\
2 \\
E\end{array}$ & $\begin{array}{l}22 \\
13 \\
25\end{array}$ & $\begin{array}{l}0.9 \\
0.5 \\
0.2\end{array}$ & - & $\dot{-}$ & $\begin{array}{c}7 \\
16 \\
15\end{array}$ & $\begin{array}{l}0.3 \\
0.6 \\
0.6\end{array}$ & $\begin{array}{l}3 \\
3 \\
3\end{array}$ & $\begin{array}{l}2.1 \\
2.1 \\
2.1\end{array}$ & $\begin{array}{l}29 \\
29 \\
40\end{array}$ & $\begin{array}{l}0.6 \\
0.6 \\
0.8\end{array}$ & $\begin{array}{l}3 \\
3 \\
3\end{array}$ & $\begin{array}{l}1.3 \\
1.3 \\
1.3\end{array}$ & $\begin{array}{l}32 \\
32 \\
43\end{array}$ & $\begin{array}{l}0.6 \\
0.6 \\
0.9\end{array}$ \\
\hline November & $\begin{array}{l}1 \\
2 \\
\mathrm{E}\end{array}$ & $\begin{array}{l}51 \\
18 \\
54\end{array}$ & $\begin{array}{l}2.1 \\
0.7 \\
2.3\end{array}$ & $:$ & $\dot{-}$ & $\begin{array}{c}13 \\
8 \\
10\end{array}$ & $\begin{array}{l}0.5 \\
0.3 \\
0.4\end{array}$ & : & : & $\begin{array}{l}64 \\
26 \\
64\end{array}$ & $\begin{array}{l}1.3 \\
0.5 \\
1.3\end{array}$ & - & $\begin{array}{l}- \\
-\end{array}$ & $\begin{array}{l}64 \\
26 \\
64\end{array}$ & $\begin{array}{l}1.3 \\
0.5 \\
1.3\end{array}$ \\
\hline December & $\begin{array}{l}1 \\
2 \\
\mathrm{E}\end{array}$ & $\begin{array}{l}15 \\
20 \\
29\end{array}$ & $\begin{array}{l}0.6 \\
0.8 \\
1.2\end{array}$ & $\begin{array}{l}1 \\
2 \\
-\end{array}$ & $\begin{array}{l}1.1 \\
2.2 \\
-\end{array}$ & $\begin{array}{c}7 \\
13 \\
18\end{array}$ & $\begin{array}{l}0.3 \\
0.5 \\
0.7\end{array}$ & : & : & $\begin{array}{l}22 \\
33 \\
47\end{array}$ & $\begin{array}{l}0.5 \\
0.7 \\
1.0\end{array}$ & $\begin{array}{l}1 \\
2 \\
-\end{array}$ & $\begin{array}{l}0.4 \\
0.9 \\
-\end{array}$ & $\begin{array}{l}23 \\
35 \\
47\end{array}$ & $\begin{array}{l}0.4 \\
0.7 \\
0.9\end{array}$ \\
\hline Total & $\begin{array}{l}1 \\
2 \\
\mathrm{E}\end{array}$ & $\begin{array}{c}1,436 \\
533 \\
403\end{array}$ & $\begin{array}{l}60.5 \\
22.4 \\
17.0\end{array}$ & $\begin{array}{c}63 \\
22 \\
4\end{array}$ & $\begin{array}{r}70.8 \\
24.7 \\
4.5\end{array}$ & $\begin{array}{c}1,113 \\
979 \\
338\end{array}$ & $\begin{array}{l}45.8 \\
40.2 \\
13.9\end{array}$ & $\begin{array}{c}59 \\
78 \\
5\end{array}$ & $\begin{array}{r}41.5 \\
54.9 \\
3.5\end{array}$ & $\begin{array}{c}2,549 \\
1,512 \\
741\end{array}$ & $\begin{array}{l}53.0 \\
31.4 \\
15.4\end{array}$ & $\begin{array}{c}122 \\
100 \\
9\end{array}$ & $\begin{array}{r}52.8 \\
43.3 \\
3.9\end{array}$ & $\begin{array}{c}2,671 \\
1,612 \\
750\end{array}$ & $\begin{array}{l}53.1 \\
32.0 \\
14.9\end{array}$ \\
\hline $\mathbf{T}$ & & 2,372 & 99.9 & 89 & 100.0 & 2,430 & 99.9 & 142 & 99.9 & 4,802 & 99.8 & 231 & 100.0 & 5,033 & 100.0 \\
\hline ES - Exp & it & Sta & & $\mathbf{S}$ & & 10n & trap & & & & & & & & \\
\hline
\end{tabular}

$85.1 \%$ of the total adults caught during the period, while $14.9 \%$ was obtained on the open land (Shan E).

In the partially disturbed environment of the GA site, the distribution pattern was quite irregular and no significant difference was observed.

Domiciliary environment.- Through the use of human bait, a total of 758 females mosquitoes was caught of which $226(29.8 \%)$ belonged to $C x$. ribeirensis and $532(70.2 \%)$ to $C x$. sacchettae.
With respect to collection sites the results were as follows (Tables 1 and 4):

Indoor Outdoor Total

CX. nibeirensis $\quad 92(26.3 \%) \quad 134(32.8 \%) \quad 226(29.8 \%)$ CX. sacchettae $258(73.7 \%) \quad 274(67.2 \%) \quad 532(70.2 \%)$

Total $\quad 350 \quad \frac{408}{758}$


Table 3 - Monthly distribution of female Culex ribeirensis and $C x$. sachettae collected in Shannon trap in the partially disturbed environment (GA), August 1992 through December 1993.

\begin{tabular}{|c|c|c|c|c|c|c|c|}
\hline & \multirow[b]{2}{*}{ Months } & \multicolumn{2}{|c|}{ Cx. ribeirensis } & \multicolumn{2}{|c|}{ Cx. sacchettae } & \multicolumn{2}{|c|}{ Total } \\
\hline & & n & $\%$ & $n$ & $\%$ & $\mathrm{n}$ & $\%$ \\
\hline \multicolumn{8}{|l|}{1992} \\
\hline & August & 73 & 18.0 & 2 & 1.2 & 75 & 13.2 \\
\hline & September & 141 & 34.8 & 3 & 1.8 & 144 & 25.4 \\
\hline & October & 5 & 1.2 & 3 & 1.8 & 8 & 1.4 \\
\hline & November & 16 & 3.9 & 24 & 14.8 & 40 & 7.1 \\
\hline & December & - & - & 58 & 35.8 & 58 & 10.2 \\
\hline \multicolumn{8}{|l|}{1993} \\
\hline & January & 15 & 3.7 & 9 & 5.6 & 24 & 4.2 \\
\hline & February & 1 & 0.2 & 2 & 1.2 & 3 & 0.5 \\
\hline & March & 25 & 6.2 & 20 & 12.3 & 45 & 7.9 \\
\hline & April & 40 & 9.9 & 11 & 6.8 & 51 & 9.0 \\
\hline & May & 6 & 1.5 & - & - & 6 & 1.0 \\
\hline & June & 4 & 1.0 & 1 & 0.6 & 5 & 0.9 \\
\hline & July & 5 & 1.2 & 1 & 0.6 & 6 & 1.0 \\
\hline & August & 45 & 11.1 & 10 & 6.2 & 55 & 9.7 \\
\hline & September & 6 & 1.5 & 5 & 3.1 & 11 & 1.9 \\
\hline & October & 1 & 0.2 & 3 & 1.8 & 4 & 0.7 \\
\hline & November & 20 & 4.9 & . & - & 20 & 3.5 \\
\hline & December & 2 & 0.5 & 10 & 6.2 & 12 & 2.1 \\
\hline Total & & 405 & 99.8 & 162 & 99.8 & 567 & 99.7 \\
\hline
\end{tabular}

GA - "Galiléia Farm"

Table 4 - Monthly distribution of female Culex ribeirensis and $C x$. sachettae collected by means of human bait in the domiciliary environment (BC), August 1992 through December 1993.

CX. ribeirensis Cx. sacchettae

Total

Months

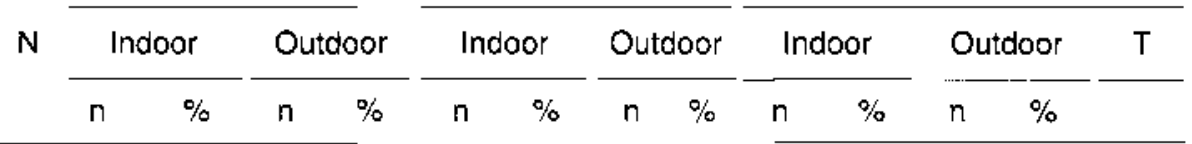

1992

$\begin{array}{lcccccccccccccc}\text { August } & 2 & - & - & 12 & 8.9 & 1 & 0.4 & - & - & 1 & 0.3 & 12 & 2.9 & 13 \\ \text { September } & 2 & 3 & 3.2 & - & - & 1 & 0.4 & 1 & 0.4 & 4 & 1.1 & 1 & 0.2 & 5 \\ \text { October } & 2 & - & - & - & - & - & - & - & - & - & - & - & - & - \\ \text { November } & 2 & 30 & 32.6 & 23 & 17.2 & 6 & 2.3 & 8 & 2.9 & 36 & 10.3 & 31 & 7.6 & 67 \\ \text { December } & 3 & 1 & 1.1 & 19 & 14.2 & 8 & 3.1 & 52 & 19.0 & 9 & 2.6 & 71 & 17.4 & 80\end{array}$

1993

\begin{tabular}{|c|c|c|c|c|c|c|c|c|c|c|c|c|c|c|}
\hline January & 2 & 14 & 15.2 & 19 & 14.2 & 5 & 1.9 & 25 & 9.1 & 19 & 5.4 & 44 & 10.8 & 63 \\
\hline February & 2 & 26 & 28.3 & 23 & 17.2 & 5 & 1.9 & 36 & 13.1 & 31 & 8.9 & 59 & 14.5 & 90 \\
\hline March & 2 & 12 & 13.0 & 10 & 7.5 & 171 & 66.3 & 23 & 8.3 & 183 & 52.3 & 33 & 8.1 & 216 \\
\hline April & 2 & 2 & 2.2 & 2 & 1.5 & 32 & 12.4 & 55 & 20.0 & 34 & 9.7 & 57 & 14.0 & 91 \\
\hline May & 2 & 1 & 1.1 & 2 & 1.5 & 23 & 8.9 & 42 & 15.3 & 24 & 6.9 & 44 & 10.8 & 68 \\
\hline June & 3 & - & - & 2 & 1.5 & 2 & 0.8 & 3 & 1.1 & 2 & 0.6 & 5 & 1.2 & \\
\hline July & 2 & 1 & 1.1 & 1 & 0.7 & 1 & 0.4 & 5 & 1.8 & 2 & 0.6 & 6 & 1.5 & 8 \\
\hline August & 2 & 1 & 1.1 & - & 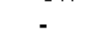 & - & 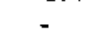 & & - & 1 & 0.3 & 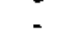 & 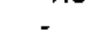 & 1 \\
\hline September & 1 & - & 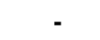 & 1 & 0.7 & - & - & 1 & 0.4 & - & - & 2 & 0.5 & 2 \\
\hline October & 2 & - & - & 7 & 5.2 & - & - & 13 & 4.7 & - & - & 20 & 4.9 & 20 \\
\hline Nov & 3 & - & - & 6 & 4.5 & 3 & 1.2 & 6 & 2.2 & 3 & 0.9 & 12 & 2.9 & 15 \\
\hline ber & 2 & 1 & 1.1 & 7 & 5.2 & - & - & 4 & 1.5 & 1 & 0.3 & 11 & 2.7 & 12 \\
\hline & 34 & 92 & 100.0 & 134 & 100.0 & 258 & 100.0 & 27 & 99.8 & 350 & 180.2 & 408 & 100.0 & 758 \\
\hline
\end{tabular}

$\mathrm{N}=$ Number of catches

$B C=$ "Barra do Capinzal" Farm 
The Williams' general means values were as follows:

$\begin{array}{lcc} & \text { Indoor } & \text { Outdoor } \\ \text { Cx. ribeirensis } & 0.46 & 0.53 \\ \text { Cx. sacchettae } & 0.50 & 0.57\end{array}$

The individual values applied for the estimation of the Williams' means were compared by the Mann-Witney text, which showed the absence of differences, at the level of $5.0 \%$, between $C x$. ribeirensis and $C x$. sacchettae.

Thus biting females of both species may be caught in the domiciliary environment, with similar frequencies (Table 4). As a general feature the peak of abundance corresponded to the December-April period when 540 ( $71.2 \%$ of the total) females were collected. That peak roughly corresponds to that obtained through the Shannon trap sampling (Table 2) in the ES area when $3,566(74.2 \%$ of the total) females were collected during the February-April period.

Synanthropy - As has already been mentioned the three environments studied were compared by means of the synanthropic indices (s) for both the mosquito species collected in these habitats. The results obtained were as follows:

$\begin{array}{lcccc} & \mathrm{a}(\%) & \mathrm{b}(\%) & \mathrm{c}(\%) & \mathrm{s} \\ \text { Cx. ribeirensis } & 7.5 & 26.9 & 65.6 & -44.7 \\ \text { Cx. sacchettae } & 17.0 & 16.0 & 67.0 & -42.0\end{array}$

Considering the general overview of this man-made environment, both mosquitoes appeared, in large measures, to retain their sylvatic habits.

Regarding the synanthropic ratios (sr) the overall value in the ES area, related to the remnant forest samples (Shan 1 and Shan 2) was 0.35 suggesting that these residual patches retain a greater number of both mosquitoes than the open land (Shan E) and the domiciliary environment (BC). Then, relating these latter two, the ratio obtained was 1.01 , which shows no overall differences between these environments as regards the two culicids. Nevertheless, when the two species were considered separately the ratios obtained were the following:
Cx. ribeirensis

$$
\begin{gathered}
\mathrm{sr}=\frac{\operatorname{Shan} \mathrm{E}}{\mathrm{Shan} 1+\text { Shan } 2}=0.20 \\
\mathrm{sr}=\frac{\mathrm{BC}}{\operatorname{Shan} \mathrm{E}}=0.56
\end{gathered}
$$

Cx. sacchettae

$$
\begin{gathered}
\mathrm{sr}=\frac{\operatorname{Shan} \mathrm{E}}{\operatorname{Shan} 1+\mathrm{Shan} 2}=0.15 \\
\mathrm{sr}=\frac{\mathrm{BC}}{\operatorname{Shan} \mathrm{E}}=1.55
\end{gathered}
$$

Thus, in the domiciliary environment (BC) $C x$. sacchettae showed a higher synanthropy than $C x$. ribeirensis when compared with the open land (Shan E) sampling.

\section{Discussion}

In a first attempt at an interpretation of the results obtained by the present research, the prevalence of $C x$. ribeirensis and $C x$. sacchettae among the Spissipes Section members must be considered. This fact was already noticed in previously published papers (Forattini et al. 5,6,13,14, 1986, 1993). Nevertheless this pattern must be taken as regional because in others regions of the Ribeira Valley others species may be prevalent. This happens in the Iguape area where $C x$. delpontei, Cx. ocossa and Culex VR, jointly with $C x$. ribeirensis and $C x$. sacchettae, showed a high frequency in the amount collected (Forattini et al. ${ }^{9}, 1990$ ).

The main objective was to compare the adult abundance at the several sites. Two different techniques for sampling were employed, Shannon traps in the ouside environment and human bait in the domicilary one. This different aspects maybe influenced the results. However, according to the local circunstances, human bit was the more suitable sampling method that was used in dwellings.

The two residual patchy woods (Shan 1 and Shan 2) in the ES area accounted for the majority of the specimens of the two mosquito species caught. Jointly with the ES open land (Shan E), the monthly distribution showed a peak abundance corresponding to the February-April period. That corresponded to the results already reported (Forattini et al. ${ }^{13}, 1993$ ) for Cx. ribeirensis caught at the same place. When the two environments are compared it would seem that the remnant forest represents something like an area of concentration from which adults of the two culicids disperse and reach the open land, seeing only immature stages 
of $C x$, ribeirensis, through as meaningless numbers, were found at the rice ES irrigation system (Forattini et al. ${ }^{12}$, 1993).

As regards biting behavior in the domiciliary environment, the general values of Williams mean showed no difference as between the two species, December-April being the period of peak abundance roughly corresponding with that at the outside ES areas. Nevertheless within the same period secondary peaks of $C x$. sacchettae were observed regarding that remarkably appeared in high numbers in the March samples (Table 4). It is hard to find an adequate explanation for this, but perhaps, besides the human bait, peculiar conditions related to the attractiveness of the increased number of fowl kept there may contribute to explain it. As regards Cr. ribeirensis, a more regular behavior in this environment was observed, in accordance with the general pattern already described for the natural outside environment (Forattini and Gomes ${ }^{7}, 1988$ ).

Regarding the degree of synanthropy of these two mosquitoes, both indices suggest weak tendencies to adapt to the domiciliary environment. This seemingly disagrees with the estimate in relation to the primitive environment for which indices of +63.0 for $C x$. ribeirensis and -3.0 for $C x$. sacchettae were obtained (Forattini et al. ${ }^{13}$,

\section{Peferences}

1. CALISHER, C.H; KINNEY, R.M; LOPES. O. de S.; TRENT. D.W; MONATH, T.P.: FRANCY, D.B. Identification of a new: Venezuelan equine encephalitis virus from Brazil. Am.JTrop.Med.Hug. 31:1260-72. 1982.

2. CALISHER. C.H.: COIMBRA, T.L.M.: LOPES, O.de S.: MLTH, D.J.: SACCHETTA, L. de A.; FRA.YCY, D.B.: LAZLICK, J.S.; CROPP, C.B. Identification of new Guama and group C serogroup Bunyaviruses and an ungrouped virus from southern Brazil. AmJ.Trop.Med.Hyg. 32:424-31, 1983.

3. CALISHER, C.H.; MONATH, T.P, MITCHELL, C.J.: SABATTINT, M.S.: CROPP: C.B.; KERSCHNER, J.; HUNT. A.R.; LAZLTCK, J.S, Artovirus investigations in Argentina, 1977-1980. IIl.Identification and characterization of viruses isolated. including new subtypes of Westem and Venezuelan Equine Encephalitis Viruses and four new Bunyaviruses (Las Maloyas, Resistencia. Barranqueras, and Antequera). AmJ.Trop.Med.Hyg. 34:956-65, 1985.

4. FORATITAI, O.P.; GOMES. A de C.: SANTOS. J.L.F.: GALATI, E.A.B; RABELLO, E.X.; NATAL, D. Observaçōes sobre atividade de mosquitos Culicidae em mata residual no vale do Ribeira.
1993). Nevertheless the comparisons were made in the light of the human dominated environment as a whole. Indeed the anthropic one studied here included several different features each one with its own characteristics. Thus the remnant forests. the cultivated open land and the dwelling houses represente quite distinct aspects of the man-made organized environment. As was mentioned above the patches of residual forest may fulfil both the preserving and the concentration roles for these mosquito populations. So it is quite understandable that they retained their sylvatic behavior when compared with their behavior in others places. Despite the comparison of their synanthropic ratios (sr), it became clear that, relating the open land to the domiciliary environment, the two culicids showed a higher frequency in the latter. $C x$ sacchettae at least seems to have preferred the houses over the open land. As a general aspect. both species have showed a clear tendency towards domiciliation (Forattini et al. ${ }^{8}, 1989$ ).

In conclusion, the present research permits to consider $C x$. ribeirensis and $C x$. sacchettae as potentially synanthropic, at least in the Ribeira Valley region. As their competence for virus transmission has been reported, it is justifiable to pay attention to these mosquitoes in the anthropic environment.

S.Paulo, Brasil. Ret:Sande Priblica, 15:557-86. 1981.

5. FORATTINI. O.P.: GOMES, A. de C.: NATAL, D.: SANTOS, J.L.F. Observaçòes sobre atividade de mosquitos Culicidae em mata primitiva da encosta no Vale do Ribeira, Sào Paulo, Brasil Rev Satide Pliblica, 20:1-20, 1986.

6. FORATTINI. O.P.: GOMES. A de C.: NATAL. D.: SAXTOS, J.L.F. Observaçóes sobre arividade de mosquitos Culicidae em matas primitivas da planície e perfís epidemiológicos de vários ambientes no Vale do Ribeira. Sào Paulo. Brasil. Ret. Salide Püblica. 20:178-203. 1986a.

7. FORATTINI, O.P. \& GOMES, A. de C. Biting activity patterns of Culex (Melanoconion) ribeirensis in southern Brazil J.Am.Mosq. Control Assoc., 4:175-8. 1988.

8. FORATTINI, O.P.: GOMES, A de C.: YATAL, D.: KAKITAYI, I.: MARLCCI, D. Preferências alimentares e domiciliaçào de mosquitos Culicidae no Vale do Ribeira, Sào Paulo. Brasil com especial referência a Aedtes scapularis e a Culex (Melanoconion). Rev'Saüde Priblica. 23:9-19, 1989.

9. FORATTINI. O.P.: GOMES, A. de C.: SANTOS, J.L.F.; KAKITA.VI. I.: MARLCCI. D. Freqüência ao ambiente humano e dispersão de nosqui- 
tos Culicidae em áreas adjacente à mata atlântica primitiva da planície. Rev. Saude Pública, 24:101-7, 1990.

10. FORATTINI, O.P.; GOMES, A. de C.; KAKITANI, I.; MARUCCI, D. Observaçōes sobre domiciliaçào de mosquitos Culex (Melanoconion) em ambiente com acentuadas modificaçóes antrópicas. Rev. Saúde Pública, 25:257-66, 1991.

11. FORATIINI, O.P.; KAKITANI, I.; MASSAD, E.; MARLCCI, D. Studies on mosquitoes (Diptera: Culicidae) and anthropic environment. 1 - Parity of blood seeking Anopbeles (Kerteszia) in South-Eastern Brazil. Rev. Saude Pública, 27:1-8, 1993.

12. FORATTINI, O.P.; KAKITANI, I.; MASSAD, E.; MARUCCI, D. Studies on mosquitoes (Diptera: Culicidae) and anthropic environment. 2 - Immature stages research at a rice irrigation system location in South-Eastern Brazil. Rev, Saúde Pública, 27:227-36. 1993.

13. FORATIINI, O.P.; KAKITAVI, I.; MASSAD, E; MARLCCI, D. Studies on mosquitoes (Diptera: Culicidae) and anthropic environment. 3 - Survey of adult stages at the rice irrigation system and the emergence of Anopbeles albitarsis in SouthEastern Brazil. Rev. Sauide Pública, 27:313-25, 1993.

14. FORATTINI, O.P.; KAKITANI, I.: MASSAD, E; MARUCCI, D. Studies on mosquitoes (Diptera: Culicidae) and anthropic environment. 4 - Survey of resting adults and synanthropic behavior in South-Eastern Brazil. Rev. Saúde Pública, 27:398-411, 1993.

15. FORATTINI, O.P.; KAKITANI, I.; MASSAD, E; MARLCCI, D. Studies on mosquitoes (Diptera: Culicidae) and anthropic environment. 7 Behaviour of adults Nyssorbynchus anophelinae with special reference to Anopbeles albitarsis 1.s, in South-Eastem Brazil. Rev. Saüde Publica, 29: 20-6,1995.
16. HADDOW, A.J. Studies on the biting-habits of African mosquitoes and appraisal of methods employed, with special reference to the twenty-four-hour catch. Bull.Entomol.Res. 45:199-242, 1954

17. HADDOW, A.J. Studies on the biting-habits and medical importance of east African mosquitoes in the genus Aedes. I- Subgenera Aedimorpbus, Banksinella and Dunnius. Bull. Entomol.Res, 50:759-79, 1960.

18. HANNAH, L:; LOHSE, D.; HUTCHINSON, C.; CARR, J.L.; LANKERANI, A. A preliminary inventory of human disturbance of World ecosystems. Ambio, 23:246-250, 1994.

19. IVERSSON, L.B. Rocio encephalitis. In: Monath, T.P., ed. - The arbovinises: epidemiology and ecology. Boca Raton. CRC Press, 1988, vi4; p. $77-92$.

20. MITCHELL, C.J.; MONATH, T.P.; SABATTINI, M.S.; CROPP, C.B.; DAFFNER, J.F.; CALISHER, C.H.; JAKOB, W.L.; CHRISTENSEN, H.A. Arbovirus investigations in Argentina, 1977. 1980. II. Arthropod collections and virus isolations from argentine mosquitoes. AmJ.Trop.Med.Hyg., 34:945-55, 1985.

21. MITCHELL, C.J.; MONATH, T.P.; SABATTINI, M.S.; DAFFNER, J.F.; CROPP, C.B.; CALISHER, C.H.; DARSIE, Jr. R.F, JAKOB, W.I. Arbovirus isolations from mosquitoes collected during and after the 1982-1983 epizootic of Western Equine Encephalitis in Argentina. AmJ.Trop.Med.Hyg., 36:107-13, 1987.

22. NLORTEVA, P. Synanthropy of blowflies (Dopt. Calliphoridae) in Finland. Ann. Entomol.Fenn, 29:1-49, 1963.

23. POVOLYY, D. Synanthropy. In: Greeberg, B., ed. Flies and disease. Princeton, Princeton Lniv, Press, 1971, v.1.

24. SALLLM, M.A.M. Revisão da Seção Spissipes de Culex (Melanoconion) (Diptera: Culicidae). São Paulo, 1994. [Tese de Doutoramento - Faculdade de Saúde Pública da USP].

\section{Resumo}

Relatam-se os resultados obtidos mediante a coleta reguiar de adultos representantes da Seçāo Spissipes de Culex (Melanoconion) realizada em ambiente antropico do Vale do Ribeira, SP, Brasil, no periodo de agosto de 1992 a dezembro de 1993. Foi possivel evidenciar a dominancia de Culex ribeirensis $\theta$ de Cx. sacchettae se bem que, no total, tenham sido identificadas dez espécies desse grupo. Esses dois mosquitos revelaram clara tendéncia a freqüentar as casas onde foram coletados, tanto no intra como no peridomicflio, mediante o emprego da isca humana. No ambiente extradomiciliar, ao que parece, as matas residusis desempenhariam o papel de concentraçáo dessas populaçōes, de onde os adultos sairiam para o terreno aberto cultivado e alcançariam as moradias. Como a competéncia vetora dessas espécies tem sido revelada mediante o isciamento de virus em condiços naturais, é de se recomendar que mereça atençăo devida, a presença desses mosquitos no ambiente artiflcial humano.

Culicidae. Ecologia de vetores. Culex. 